sist of microfibrils with extreme aspect ratios. The microfibular structure can be highly complex consisting of regions of variable crystallinity and polymorphic. ${ }^{7,8}$

In this presentation, the authors have applied the new tools $^{1-6}$ to this old problem. This includes the determination of the crystalline polymorphs and the amorphous structure via methods described in references 1 and 2. Subsequently, cluster analysis, crystallite size simulation and pattern matching was applied to study pulp and paper as well as pharmaceutical tablets.

${ }^{1}$ J. A. Kaduk and P. Langan, "Crystal structures and powder patterns of celluloses I $\alpha, \mathrm{I} \beta$, and II," Private Communication. To be published. Earlier results presented at PPXRD-2, 2002. Abstract available at www.icdd.com/ppxrd/02.

${ }^{2}$ S. Bates, "Analysis of Short Range Order in X-ray Amorphous Indomethacin Using X-ray Powder Diffraction and Pair-Wise Distribution Function," PPXRD-3, February 2004. Abstract available at www.icdd.com/ppxrd/03 and a related presentation at www.icdd.com/ppxrd/05.
${ }^{3}$ T. Degan, "XRPD Pattern Matching: Probability Based Versus Image Comparison,” PPXRD-5, 2006. Abstract available at www.icdd.com/ppxrd/05.

${ }^{4}$ C. Gilmore, "The Search for Polymorphs and Salts Using High Throughput Powder Diffraction, Spectroscopy and Other Techniques," PPXRD-4, 2005. Abstract available atwww.icdd.com/ppxrd/02.

${ }^{5}$ T. G. Fawcett and S. N. Kabekkodu, "Mapping Polymorphs by Combining Cluster Analysis with Diffraction Databases," PPXRD-5, 2006. Abstract available at www.icdd.com/ppxrd/ 05.

${ }^{6}$ P. Scardi, M. Leoni, and J. Faber, "Diffraction line profile from disperse system: A simple alternative to Voigtian profiles," Powder Diffraction, 21, No. 4, 2006.

${ }^{7}$ R. H. Atalla and B. L. Vander Hart, 1984, Science, 223, 283. ${ }^{8}$ A. A. Baker, W. Helburt, J. Sugiyama, and M. J. Miles, "New Insight into Cellulose Structure by Atomic Force Microscopy Shows I $\alpha$ Crystal Phase at Near Atomic Resolution,” Biophysical Journal, 79, 2000, pp. 1139-1145.

\title{
Calendar of Meetings
}

\author{
Donald R. Petersen \\ Greenleaf Associates \\ 6210 Siebert Street \\ Midland, MI 48640-2724, USA \\ drpetersen@ voyager.net
}

\section{2-7 June 2007}

44th Annual Meeting of the Clay Minerals Society. Santa $\mathrm{Fe}$, New Mexico, USA. Includes the workshop Carbon Stabilization by Clays in the Environment: Process and Characterization Methods. [Contact: Info: http://www. sandia.gov/clay].

\section{7-21 June 2007}

2nd International Conference on Archaeometallurgy in Europe. Grado and Aquileia, Italy. [Contact: Associazione Italiana di Metallurgia, Piazzale Rodolfo Morandi 2, I-20121 Milano, Italy. Tel: 39 (02) 7602 1132; Fax: 39 (02) 7602 0551; E-mail: aim@aimnet.it; Info: http://www. aimnet.it/allhtml/archaeometallurgy2.htm].

\section{5-29 June 2007}

4th European Conference on Neutron Scattering. Lund, Sweden. [Contact: ECNS 2007, c/o Tommy Nylander, Physical Chemistry 1, Lund University, Post Office Box 124, SE22100 Lund, Sweden. Info: http://www.ecns2007.org].

\section{5-30 June 2007}

Combined Societies' Meeting-Mineralogical Society of Great Britain and Ireland, Mineralogical Society of America, and Mineralogical Society of Canada. Cambridge, England, UK. [Contact: M. A. Carpenter at mc43@esc.cam.ac.uk; Info: http://www.minersoc.org/pages/ meetings/frontiers/index.html].

\section{8-13 July 2007}

XVIII International Conference on the Chemistry of the
Organic Solid State. Mérida, Venezuela. [Contact: Prof. Graciela Díaz de Delgado, Laboratorio de Cristalografia, Facultad de Ciencias, Universidad de Los Andes, Mérida 5101, Venezuela. E-mail: diaz@ula.ve; Info: http://www.ula. ve/eventos/iccoss].

\section{9-13 July 2007}

59th Annual Meeting: Inter/Micro Conference. Chicago, Illinois, USA. Sponsored by the McCrone Research Institute. [Contact: McCrone Research Institute, 2820 South Michigan Avenue, Chicago, IL 60616-3292 USA. Tel: 1 (312) 842 7100; Fax: 1 (312) 842 1078; E-mail: info@mcri.org; Info: http://www.mcri.org/IM_info_page.html].

21-26 July 2007

American Crystallographic Association Annual Meeting. Salt Lake City, Utah, USA. Local Chair, Christopher Hill; Program Chair, Jill Trewhella. [Info: http//www. AmerCrystalAssn.org].

\section{2-27 July 2007}

Euroclay 2007. Aveiro, Portugal. [Contact: Prof. Celso Gomes at cgomes@geo.ua.pt; Info: http://www.ing.pan.pl/ecga _js/ecga_PDF/euroclay_07.pdf].

\section{3-31 July 2007}

ICNX 2007 International Conference on Neutron and $\mathrm{X}$-ray Scattering. Serpong and Bandung, Indonesia. [Contact: Edy Giri R. Putra, Neutron Scattering Laboratory, National Nuclear Energy Agency (BATAN), Gedung 40, Kawasan Puspiptek Serpong, Tangerang 15314, Indonesia. 
Tel: $62 \quad$ (21) 756 6727; Fax: 62 (21) 756 0926; E-mail: icnx2007@centrin.net.id; Info: http://centrin.net.id/ $\sim$ nslbatan/icnx].

\section{July-3 August 2007}

15th International Conference on Vacuum Ultraviolet Radiation Physics. Berlin, Germany. "For those working in the field of developing synchrotron, laser, or plasma-based VUV and soft X-ray sources, and applying photons in this spectral range." [Contact: Wolfgang Eberhardt, BESSY $\mathrm{mbH}$, Albert-Einstein-Strasse 15, 12489 Berlin, Germany. Tel: 49 (30) 6392 4710; Fax: 49 (30) 6392 2989; E-mail: vuvxv@bessy.de; Info: http://www.bessy.de/VUVXV/front _content.php].

\section{July-3 August 2007}

56th Annual Denver X-ray Conference. Colorado Springs, Colorado, USA. Sponsored by the International Centre for Diffraction Data. Includes workshops (30-31 July) on X-ray Optics; Neutron Scattering for Characterization; Twodimensional XRD; Diffraction Peak Broadening and Peak Shape Analysis; Small-angler Scattering; Rietveld Applications; X-ray Crystallography without Crystals; Analysis of RoHS/WEEE Elements; XRF Sample Preparation; Basic XRF; Quantitative Analysis; Energy Dispersive XRF; and Trace Analysis. [Contact: Denise Flaherty, Conference Coordinator, ICDD, 12 Campus Boulevard, Newtown Square, PA 19073, USA. Tel: 1 (610) 325 9814; Fax: 1 (610) 325 9823; E-mail: flaherty@icdd.com; Info: http://www.dxcicdd.com].

\section{2-17 August 2007}

15th International Zeolite Conference. Beijing, China. [Contact: Prof. Shilum Qiu, State Key Laboratory of Inorganic Synthesis and Preparative Chemistry, Jilin University, Linyuan Road 1788, Changchun 130012, China. Tel: 86 (431) 5168590; Fax: 86 (431) 5168614: E-mail: izc@jlu.edu.cn; Info: http://www.15izc.org.cn].

\section{0-22 August 2007}

Conference on Mathematical and Theoretical Crystallography. Marrakech, Morocco. A satellite conference of ECM-24. [Info: http://www.lcm3b.uhp-nancy.fr/mathcryst/ marrakech2007.htm].

\section{0-22 August 2007}

Conference on Art and Crystallography: The Enchanting Crystallograph of Moroccan Ornaments. Marrakech, Morocco. A satellite conference of ECM-24. The first two days will consist of lectures; the last day will be devoted to an excursion to the Kasbah de Telouet, a site renowned for the richness of its ornaments. [Info: http://www.lcm3b.uhpnancy.fr/mathcryst/marrakech2007.htm].

\section{2-27 August 2007}

ECM-24 24th European Crystallographic Meeting. Marrakech, Morocco. Held at the Palais des Congrès. [Info: http://www.ecm24.org].

\section{9-14 September 2007}

EuroAnalysis XIV. Antwerp, Belgium. [Contact: Luc Van't Dack, Department of Chemistry, University of Antwerp, Universiteitsplein 1, B-2610 Antwerp, Belgium. Tel: 32
(3) 82023 43; Fax: 32 (3) 82023 43; E-mail: Luc.Vantdack@ua.ac.be; Info: http://www.euroanalysis xiv.ua.ac.be].

\section{9-14 September 2007}

23rd International Meeting on Organic Geochemistry. Torquay, England, UK. [Contact: Hannah Evans, IMOG 2007 Secretariat IGI Ltd., Hallsannery, Bideford, Devon EX39 5HE, UK. Tel: 44 (1237) 471 749; Fax: 44 (1237) 421 700; E-mail: secretary@imog2007.org; Info: http://www. imog2007.org/index.htm].

\section{7-21 September 2007}

Joint 21st AIRAPT and 45th EHPRG International Conference on High Pressure Science and Technology. Catania, Sicily, Italy. Held at the University of Catania. [Contact: G. G. N. Angilella, Department of Physics and Astronomy, University of Catania, Via S. Sofia 64, Catania I-95123, Italy. Fax: 39 (095) 378 5231; E-mail: info @ airapt-ehprg-2007.it; Info: http://www.aip.org/cal/viewbyuser.jsp?eid=1084].

\section{7-9 October 2007}

5th Size-Strain Conference: Diffraction Analysis of the Microstructure of Materials. Garmisch-Partenkirchen, Germany. [Info: http://www.mf.mpg.de/en/abteilungen/ mittemeijer/ss-v/welcome.htm].

\section{2-18 October 2007}

34th Annual Conference of the Federation of Analytical Chemistry and Spectroscopy Societies (FACSS). Memphis, Tennessee, USA. [Contact: Cindy Lilly, FACSS, Post Office Box 24379, Santa Fe, NM 87502, USA. Tel: 1 (505) 820 1648; Fax: 1 (505) 989 1073; E-mail: fascc@facss.org; Info: http://www.facss.org].

\section{4-7 November 2007}

Eighth Conference of the Asian Crystallographic Association. Taipei, Taiwan, R. O. C. [Contact: AsCA 2007 Secretariat, Tze-Chiang Foundation of Science and Technology, 101 Sect. 2 Kuang Fu Road, Hsinchu 30013, Taiwan. Tel: 886 (3) 5726360; Fax: 886 (3) 5716782; E-mail: asca2007@tcfst.org.tw; Info: http://www.asca2007.tw/ index.html].

\section{Spring 2008}

\section{0-14 March 2008}

International Centre for Diffraction Data, Spring Meeting. Newtown Square, Pennsylvania, USA. [Contact: Linda Shertz, international Centre for Diffraction Data, 12 Campus Boulevard, Newtown Square, PA 19073-3273, USA. Tel: 1 (610) 325 9814; Fax: 1 (610) 325 9823; E-mail: shertz@icdd.com; Info: http://www.icdd.com].

British Crystallographic Association Annual Spring Meeting. Swansea, Wales, UK. [Contact: Info: http://cryst.bbk.ac.uk/bca/meets/meets.htm].

\section{May-5 June 2008}

American Crystallographic Association Annual Meeting. Knoxville, Tennessee, USA. Local Chair, Jason Hodges, Program Chairs, Paul Butler and Dean Miles. [Info: http:// www.hwi.buffalo.edu/aca/meetingspg

_list/futuremeetings.html]. 
9-14 June 2008

10th International Conference on Quasicrystals. Zurich, Switzerland. Abstracts are invited in all areas of study including synthesis, crystal growth and structure, phase stability, surfaces and thin films, physical properties, applications, and new frontiers of quasicrystals and cluster-based complex metallic alloys. [Contact: Conference Secretariat, Mrs. Cornelia Aurelio, Laboratory of Crystallography, ETH Zurich, Wolfgang-Pauli-Strasse 10, CH-8093 Zurich, Switzerland. Tel: 41 (44) 632 3769; Fax: 41 (44) 632 1133; E-mail: aurelio@mat.ethz.ch; Info: http://icq10.ethz.ch].

\section{3-31 August 2008}

2Ist IUCr General Assembly and International Congress of Crystallography. Osaka, Japan. [Info: http://www. congre.co.jp/iucr2008].
25-30 July 2009

American Crystallographic Association Annual Meeting. Toronto, Canada. [Info: http://www.amercrystalassn.org/ meetingspg_list/futuremeetings.html].

\section{August 2009}

25th European crystallographic Meeting. Istanbul, Turkey. [Info: http://www.ecanews.org/meetings.htm].

August 2010

26th European Crystallographic Meeting. Darmstadt, Germany. [Info: http://www.ecanews.org/meetings.htm].

August 2011

22nd IUCr General Assembly and International Congress of Crystallography. Madrid, Spain.

\section{Short Courses \& Workshops}

\section{3-6 June 2007}

12th European Workshop on Metal-Organic Vapour Phase Epitaxy. Bratislava, Slovakia. [Contact: EW-MOVPE Secretary, Institute of Electrical Engineering, Slovak Academy of Sciences, 84104 Bratislava, Slovakia. Tel: 421 (2) 5477 5806; Fax: 421 (2) 5477 5816; E-mail: ewmovpe@savba.sk; Info: http://elu.sav.sk/EW-MOVPE/ index.html].

\section{4-8 June 2007}

ICDD X-ray Clinic: I. Fundamentals of X-ray Powder Diffraction. Newtown Square. Pennsylvania, USA. [Contact: Leah Mooney, Education Coordinator, ICDD, 12 Campus Boulevard, Newtown Square, PA 19073, USA. Tel: 1 (610) 325 9814; Fax: 1 (610) 325 9823; E-mail: clinics@icdd.com; Info: http://www.icdd.com/ education/clinics.htm].

\section{7-17 June 2007}

Engineering of Solid State Properties: Synthesis and Development of New Materials. Erice, Italy. The 39th crystallographic course at the Ettore Majorana Centre. The course directors are D. Braga (Bologna) and J. Novoa (Barcelona). [Info: http://www.crystalerice.org/2007.htm].

\section{1-13 June 2007}

2nd TOPAS Users' Meeting. Karlsruhe Germany. TOPAS is a system of proprietary software for the analysis of powder diffraction data. [Contact: Bruker AXS GmbH, Arnt Kern, Östliche Rheinbrückenstrasse 49, 76187 Karlsruhe, Germany. Tel: 49 (721) 595 5669; Fax: 49 (721) 595 6693; E-mail: arnt.kern@bruker-axs.de; Info: http://www. bruker-axs.de].

\section{1-15 June 2007}

ICDD X-ray Clinic: II Advanced Methods in X-ray Powder Diffraction. Newtown Square. Pennsylvania, USA. [Contact: Leah Mooney, Education Coordinator, ICDD, 12 Campus Boulevard, Newtown Square, PA 19073, USA. Tel:
1 (610) 325 9814; Fax: 1 (610) 325 9823; E-mail: clinics@icdd.com; Info: http://www.icdd.com/education/ clinics.htm].

\section{4-29 June 2007}

Ninth International Workshop on Physical Characterization of Pharmaceutical Solids. Natick, Massachusetts, USA. Held at the Crowne Plaza Boston in Natick. [Info: http://www.assainternational.com/workshops/ iwpcps_9/iwpcps_9.cfm].

\section{5-20 July 2007}

Summer School on Mathematical and Theoretical Crystallography. Havana, Cuba. [Mariela Hurtado Camero, IMRE, Universidad de La Habana Zapata entre Mazón y G, CP 10400 La Habana, Cuba. Tel: 53 (7) 8707666; Fax: 53 (7) 8794651; E-mail: mhc@imre.oc.uh.cu; Info: http://www. cristalografia.net/havana2007].

\section{July-8 August 2007}

Crystallography Summer School. La Jolla, California, USA. Held at the University of California, San Diego. Limited to 25 active research chemists, from graduate students to senior faculty members. The course is an intensive introduction (or refresher) to all aspects of inorganic crystallography from crystal growth to publication of structure data. Typically students complete two and often three structure determinations during the week-long course. [Contact: Arnold L. Rheingold, UCSD Department of Chemistry and Biochemistry, Urey Hall Room 5128, 9500 Gilman Drive, M/C 0358, La Jolla, CA 92093-0358, USA. Tel: 1 (858) 822 3870; Fax: 1 (858) 822 3872; E-mail: arheingold@ucsd.edu; Info: http://chem-tech.ucsd.edu/Recharges/SMXF/overview. html].

\section{5-17 August 2007}

Zürich Crystallography School 2007. Zürich, Switzerland. [Info: http://www.oci.uzh.ch/diversa/xtal_school]. 